Manuelle Medizin 2010 • 48:177-178

DOI 10.1007/s00337-010-0764-0

Online publiziert: 18. Juni 2010

(c) Springer-Verlag 2010

\author{
L. Beyer \\ Ärztehaus Mitte, Jena
}

\title{
Arbeitsfeld der manuellen Medizin in breiter Beachtung
}

folgten Publikationen nun für die Extremitäten. Sie beschreiben in ihrem Beitrag „manualmedizinische Syndrome, die sich teilweise in bestehende Vorstellungen wie die zu den Epikondylopathien einordnen, teilweise aber auch eigenständige Entitäten darstellen. Typische manualmedizinische Befundkonstellationen werden anhand von Leitsymptomen vorgestellt. Dabei wird die differenzialdiagnostische Abgrenzung besonders zu den neurologischen und orthopädischen Krankheitsbildern ausführlich abgehandelt. Die vorgeschlagene manualmedizinisch-osteopathische Betrachtungsweise ermöglicht differenzialdiagnostische Überlegungen jenseits von nervaler Kompression, knöcherner Degeneration oder Durchblutungsstörungen. Sie bietet die Möglichkeit einer effektiven funktionsbezogenen Behandlungsplanung und könnte im Einzelfall invasive Eingriffe ersetzen.“

1. Die manuelle Medizin ist dabei, ihr eigenes Gebiet neu zu definieren. Die bisherigen theoretischen Ansätze der Blockierung und der segmentalen Störung werden eingegliedert in systematische Ansätze von funktionellen „Verkettungen“, die aber noch sehr empirisch betrachtet werden, und in die Auffassung von einer „muskuloskeletalen Medizin“, wobei manuelle Therapie eine dominierende Stellung in einer konservativen Behandlung einnehmen soll. Ein neues Denken zeigt sich aber besonders dadurch, dass die Funktion, die Störung der Funktion und deren Symptome in den Mittelpunkt der diagnostischen und therapieplanenden Überlegungen treten. Buchmann et al. zeigen solche Überlegungen nach bereits er-

\section{(7) Die manuelle Medizin hat Einzug in viele medizinische Gebiete gehalten}

2. Die manuelle Medizin hat unumkehrbar Einzug in viele medizinische Gebiete gehalten. Das Heft enthält gleich zwei umfangreiche Berichte von Tagungen, die sich vordergründig der manuellen Medizin widmen - einmal von einer „Kinder-CMD-Konferenz" und einmal mit Blick auf ältere Menschen. Darin heißt es: „Denn nur derjenige, der weiß, welche Möglichkeiten die benachbarten Fachdisziplinen haben, kann gezielt überweisen und im interdisziplinären Verbund therapieren. Das Ziel ist, die physiologische Entwicklung des Menschen in seiner Ganzheit von frühester Kindheit an zu fördern, ohne überzutherapieren. So können wir es schaffen, funktionell ungünstige Einflüsse und Entwicklungen im Entstehen zu erkennen und mit kleinen Maßnahmen gegenzusteuern, bevor eine späte funktionelle Entgleisung resultiert, deren Ursache bei langer Leidensgeschichte nur noch schwer aufzuspüren ist. Auch liegt es im Interesse langzeitstabiler Behandlungserfolge, funktionsorientiert zu denken und zu handeln.“

Dass dies nicht nur mehr eine Tendenz ist, zeigen auch Beiträge von Henning et al. mit Bezug zum Fachgebiet der HNO und zur Pädiatrie, von Schupp, der eine interdisziplinäre Therapie in der Zahnheilkunde vorstellt, und aus einer Promotion von Matyssek, die erkennbar macht, dass auch in der Anatomie noch Neues erwartet werden kann

Und noch ein weiterer Gesichtspunkt, der auf eine neue Qualität der Verbreitung und Anerkennung schließen lässt: Es werden in letzter Zeit deutliche Tendenzen sichtbar, einzelne Teile, einzelne Aspekte der manuellen Medizin zu verselbstständigen. Dies wäre nicht in diesem Ausmaße möglich, wenn die manuelle Medizin nicht inzwischen einen hohen Stellenwert im ambulanten und klinischen Bereich erlangt hätte. Sehen wir es also als vorübergehend und positiv an, wenn Ärzte sich als „Triggerpunkttherapeuten“, „Dorn-Therapeuten“ oder „Osteopathen" um Anerkennung bemühen. Allerdings hat der Deutsche Ärztetag ge- 
fordert, „der zunehmenden Aufweichung klarer rechtlicher Zuständigkeiten bei der Ausübung von Heilkunde Einhalt zu gebieten. Diese negativen Entwicklungen gehen zulasten der Versorgungsqualität und der Patientensicherheit und verletzen haftungsrechtliche Standards. Stattdessen ist das Delegationsprinzip fortzuentwickeln und rechtssicher so auszugestalten, dass alle Bereiche der Patientenversorgung hiervon profitieren."

Ich wünsche allen unseren Lesern einen sonnigen Sommer und erholsamen Urlaub.

Vielleicht sieht man sich ja zum Kongress der DGMM am 24.September in Potsdam wieder.

\section{Mit freundlichen Grüßen}

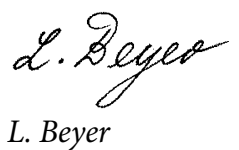

\section{Korrespondenzadresse}

\section{Prof. Dr. L. Beyer}

Ärztehaus Mitte

Westbahnhofstr. 2, 07745 Jena

LoBeyer@t-online.de

\section{September 2010}

Potsdam 16.-18.09.2010

5 gemeinsamer internationaler Kongress der DGG/ÖGGG

Geriater-Kongress „25 Jahre DGG"

Auskunft: Herr Reiner Münster,

gerikomm Media $\mathrm{GmbH}$,

Winzerstr. 9, 65207 Wiesbaden,

Fon: 061 22/70 5236 ,

Fax: 061 22/70 76 98,

reiner.muenster@t-online.de,

www.dggeriatrie.de/php/showsite

Dresden 23.-25.09.2010

44. DEGAM Kongress für Allgemeinmedizin und Familienmedizin

Auskunft: DEAGAM-Bundesgeschäftsstelle,

c/o Institut für Allgemeinmedizin

Haus $10 \mathrm{C} / 1$. Stock,

Klinikum der Johann Wolfgang Goethe-

Universität, 60590 Frankfurt,

Fon: 069-65 007245 ,

froehlich@degam.de,

www.degam2010.de

Potsdam 24.-26.09.2010

Kongress der DGMM 2010

Biomechanik - Sensomotorik - Schmerz:

drei Seiten einer Medaille

Auskunft: Deutsche Gesellschaft für Manuelle

Medizin, Ärztehaus Mitte,

Westbahnhofstr, 2, 07745 Jena,

Fon: 03641622178,

Fax: 03641622178

post@dgmm.de

Potsdam 24.-26.09.2010

DGMM-Kongress

"3 Seiten einer Medaille"

Auskunft: Frau Astrid Böhme,

Westbahnhofstr. 2, 07745 Jena

Fon: 03641 622111,

post@dgmm.de

Berlin 30.09.-02.10.2010

115. Jahreskongress der Deutschen

Gesellschaft für Physikalische Medizin

und Rehabilitation e.V.

Auskunft: Deutschen Gesellschaft für

Physikalische Medizin und Rehabilitation e.V.

Geschäftsstelle, Budapester Str. 31, 01069

Dresden,

Fon: 0351/8975932

Fax: 0351/8975939,

geschaeftsstelle@dgpmr.de

www.dgpmr.de/Kongress2010.pdf

\section{Oktober 2010}

München 05.-08.10.2010

58. Kongress der Deutschen Gesellschaft

für Gynäkologie und Geburtshilfe

Werte, Wissen,Wandel

Auskunft: Frau Anja Röder, Congress

Organisation Thomas Wiese $\mathrm{GmbH}$

Hohenzollerndamm 125, 14199 Berlin,

Fon: +49 (0) 30/85 $9962-17$,

Fax: +49 (0) 30/85 079826 ,

dggg@ctw-congress.de,

www.dggg-kongress.de
Mannheim 06.-09.10.2010

Deutscher Schmerzkongress 2010

Auskunft: Herr Manfred Müller, m:con,

Rosengartenplatz 2, 68161 München,

manfred.mueller@mcon-mannheim.de,

www.schmerzkongress2010.de

Berlin 26.-29.10.2010

Deutscher Kongress für Orthopädie und Unfallchirurgie 2010

Auskunft: Frau Ulrike Kuttler, Department

Orthopädie \& Traumatologie

Universitätsklinikum der

Alberts-Ludwigs-Universität,

Hugstetter Str. 55, 79106 Freiburg,

Fon: 0761/270 2699,

Fax: 0761/2783,

sekretariat.dot@uniklinik-freiburg.de,

www.orthopaedie-unfallchirurgie.de

\section{November 2010}

Frankfurt/Main 10.-13.11.2010

Deutscher Zahnärztetag 2010

Gemeinschaftstagung der DGZMK mit allen

DGZMK-Fachgesellschaften

Auskunft: Quintessenz Verlags-GmbH,

Komturstraße 18, 12099 Berlin

Fon: +49 (0)30 / 76180-624, -630

Fax: +49 (0)30 / 76180-693,

kongress@quintessenz.de,

www.dtzt.de

Düsseldorf 17.-20.11.2010

MEDICA

42. Weltforum der Medizin

Auskunft: MEDICA Deutsche Gesellschaft für

Interdisziplinäre Medizin e.V.,

Postfach 700149, 70571 Stuttgart,

Fon: 0711-720712-0,

Fax: 0711-720712-29,

gw@medica-ev.de,

www.medica-ev.de 